\title{
Convergent Evolution of Boats with Sails
}

\author{
A. Bejan ${ }^{1 *}$, L. Ferber ${ }^{2} \&$ S. Lorente ${ }^{3}$ \\ This article unveils the geometric characteristics of boats with sails of many sizes, covering the \\ range $10^{2}-10^{5} \mathrm{~kg}$. Data from one hundred boat models are collected and tabulated. The data show \\ distinct trends of convergent evolution across the entire range of sizes, namely: (i) the proportionality \\ between beam and draft, (ii) the proportionality between overall boat length and beam, and (iii) the \\ proportionality between mast height and overall boat length. The review shows that the geometric \\ aspect ratios (i)-(iii) are predictable from the physics of evolution toward architectures that offer greater \\ flow access through the medium.
}

Nature impresses us with images, changes and tendencies that repeat themselves innumerable times even though "similar observations" are not identical to each other. In science, we recognize each ubiquitous tendency as a distinct phenomenon. Over the centuries, our predecessors have summarized each distinct phenomenon with its own law of physics, which then serves as a 'first principle' in the edifice of science. A principle is a 'first principle' when it cannot be deduced from other first principles.

This aspect of organization in science is illustrated by the evolution of thermodynamics to its current state ${ }^{1,2}$. For example, 150 years ago the transformation of potential energy into kinetic energy and the conservation of "caloric" were fused into one statement - the first law of thermodynamics - which now serves as a first-principle in physics. It was the same with another distinct tendency in nature: everything flows (by itself) from high to low. This, the phenomenon of one-way flow, or irreversibility, was summarized in another statement at the same time - the second law of thermodynamics - which serves as another first-principle in physics.

Why do the most common occurrences need such a long time to be recognized as natural tendencies (phenomena), and even longer to be recorded in physics with a short statement, a first principle? Because the evolution of the human mind is an integral part of the evolution of the human, to adapt and survive while struck by unexpected dangers, environmental, animal, and human. The first thing that we question is the unusual (the "surprise", which means being grabbed from above, as if in the claws of a predator). Questioned the least are the most common observations, the familiar, the not threatening. This is why new questions in science are rare.

Nowhere is the human approach to science more evident than in the face of the natural phenomenon of evolutionary organization ${ }^{1,3}$. Images, morphing images, impress us constantly, yet the most common images go unnoticed. For example, the oneness of natural tree-shaped architectures of the inanimate realm (e.g., river basins) and the animate realm (e.g., human lungs, city traffic) is evident and intriguing. Recent articles are drawing attention to phenomena of evolution that are general and belong in physics ${ }^{3-23}$. This literature shows that such phenomena are predictable. Examples are the architectures of lungs ${ }^{24}$ and corals ${ }^{25}$, the life span and life travel of animals, vehicles, rivers and the winds ${ }^{26}$, the round cross sections of all jets and plumes ${ }^{27}$, the dendritic architecture and S-shaped history of dendritic solidification such as snowflakes ${ }^{28}$, the arrow of time of evolutionary organization ${ }^{29}$, and the fact that humans prefer unwittingly certain shapes and proportions, from the shapes of the Egyptian pyramids $^{30}$ and the shapes of fires (piles of fuel $)^{31}$, to the golden-ratio shape of drawings, images and text.

Because of its physics basis, the phenomenon of evolution can be imagined the way it happened, in retrospect. Further ahead along this line, evolution can be not only predicted but also witnessed in our life time, for example, by observing technological evolution. The geometric similarity of modern commercial aircraft ${ }^{32}$, like the similarity of helicopters ${ }^{33}$ and automobiles ${ }^{34}$, shows that human movement on the world map is facilitated by the generation and persistence of certain shapes and structures.

In this paper we strengthen this message by questioning an evolutionary phenomenon of technology that is evident (Fig. 1) but goes unquestioned. Why do boats with sails look the same? They have sails that are roughly as tall as the length of the hull. They have hulls that are longer than they are wide. Furthermore, they are submerged

${ }^{1}$ Duke University, Department of Mechanical Engineering and Materials Science, Box 90300, Durham, NC, 277080300, USA. '2 Lockheed Martin Space, 12257 South Wadsworth Boulevard, Littleton, CO, 80125, USA. ${ }^{3}$ Villanova University, Department of Mechanical Engineering, Villanova, PA, 19085, USA. *email: abejan@duke.edu 

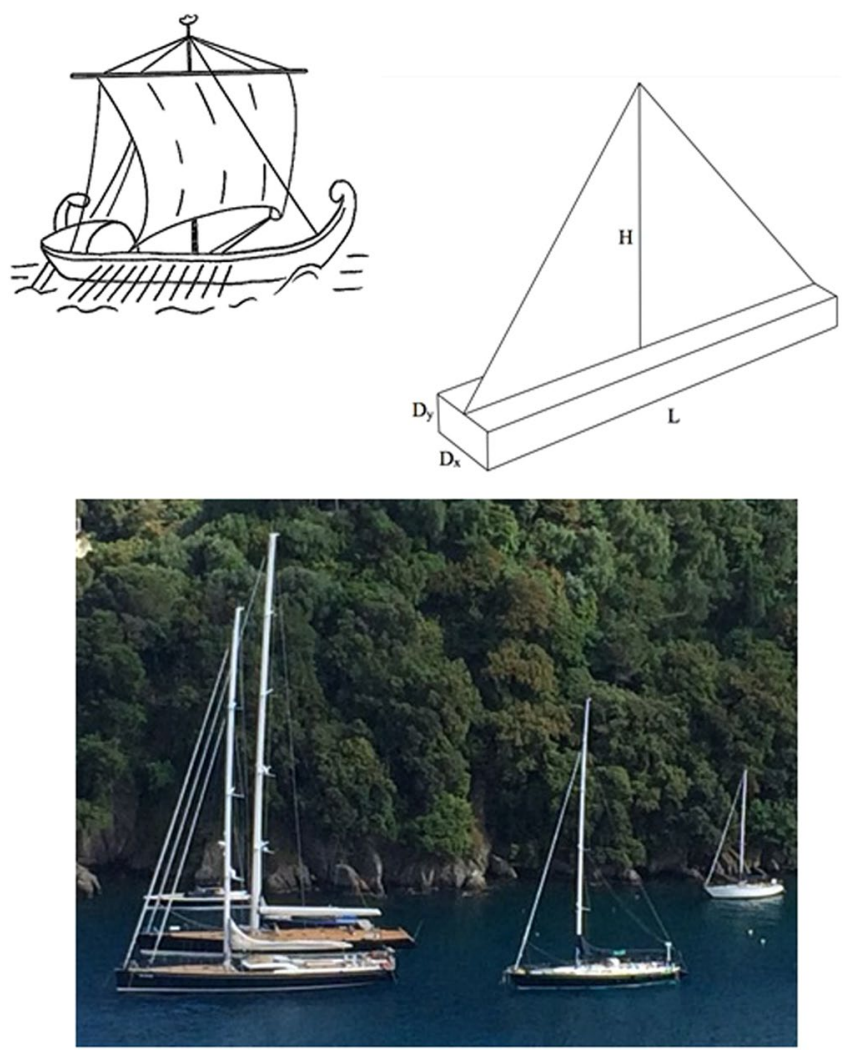

Figure 1. The geometric similarity of boats with sails, clockwise: ancient Egyptian galley, the essential length scales of the moving body, and modern sailboats (photo: Adrian Bejan).

to a depth that is greater when the hull is wider. The boat has been this way since antiquity. Even more intriguing is that the large boat looks just like the small boat. Why?

\section{Theory}

The reason for all these observations is the human tendency to move more easily on earth ${ }^{1,3}$. The vehicle architecture that emerges is a reflection of the urge of all its builders and users to move more easily, to have greater access to the surroundings. In recorded times, this tendency gave birth to artifacts (vehicles) in which people encapsulate themselves to acquire greater access. From this idea, the convergent evolution of all boats with sails is deducible.

The boat moves horizontally with the speed $V_{w}$ on the surface of the water. The wind with the speed $V_{a}$ engages the sail with the force,

$$
\mathrm{F}_{\mathrm{a}} \sim \mathrm{C}_{\mathrm{D}}(\mathrm{HL} / 2) \frac{1}{2} \rho_{\mathrm{a}} \mathrm{V}_{\mathrm{a}}^{2}
$$

where $C_{D} \sim 1$ is the drag coefficient, $\rho_{a}$ is the air density, and (HL/2) is the sail area: $\mathrm{H}$ is the height of the mast, and $\mathrm{L}$ is the hull length. The driving force $\mathrm{F}_{\mathrm{a}}$ is matched by the drag force experienced by the hull against the water,

$$
\mathrm{F}_{\mathrm{w}} \sim\left[\mathrm{C}_{\mathrm{D}} \mathrm{D}_{\mathrm{x}} \mathrm{D}_{\mathrm{y}}+\mathrm{C}_{\mathrm{f}}\left(\mathrm{D}_{\mathrm{x}}+2 \mathrm{D}_{\mathrm{y}}\right) \mathrm{L}\right] \frac{1}{2} \rho_{\mathrm{w}} \mathrm{V}_{\mathrm{w}}^{2}
$$

where $D_{x}$ is the hull width, $D_{y}$ is the depth of the submerged portion of the hull, $\rho_{w}$ is the water density, and $C_{f}$ is the skin friction coefficient for turbulent flow, the order of magnitude of which is $\mathrm{C}_{\mathrm{f}} \sim 10^{-235}$. Note the two terms in the square brackets: the first accounts for the drag experienced by the hull frontally, as a blunt body, and the second is due to the fluid friction along the hull. As we show later in the discussion of Fig. 2, the forces that propel the boat can vary depending on the angle of attack.

From the balance between $F_{w}$ and $F_{a}$ emerges the ratio $V_{w} / V_{a}$, which is larger when the quantity in square brackets in Eq. (2) is smaller. This quantity varies in accord with the two aspect ratios of the configuration, $D_{x} / D_{y}$ and $D_{x} / L$, subject to the displaced volume of water $\left(D_{x} D_{y} L\right)$, which is fixed because it is dictated by the total weight of the boat. It is easy to show analytically that the quantity in the square brackets in Eq. (2) is minimum when

$$
\frac{D_{x}}{D_{y}} \sim 2
$$



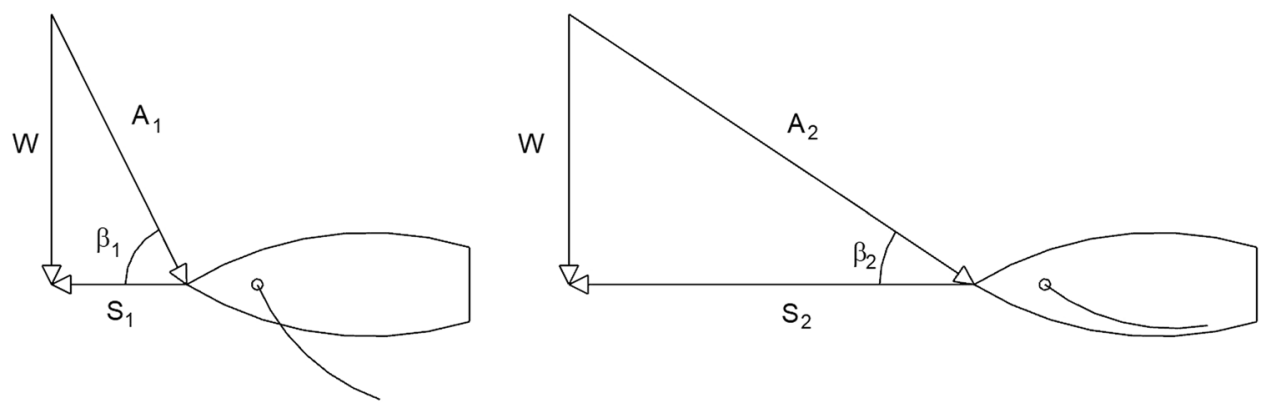

Figure 2. Two cases showing the relationship between wind speed (W), apparent wind speed (A), boat speed $(S)$ and apparent wind angle $(\beta)$. The sails are trimmed to account for apparent wind angle.

and

$$
\frac{\mathrm{L}}{\mathrm{D}_{\mathrm{x}}} \sim \frac{\mathrm{C}_{\mathrm{D}}}{2 \mathrm{C}_{\mathrm{f}}}>1
$$

This settles the question of the shape of the hull and that of most fish: they should be slender in profile, and relatively round when viewed in cross section.

What about the shape of the sail? When the aspect ratios of Eqs. $(3,4)$ apply, the drag force in the water [Eq. (2)] becomes

$$
\mathrm{F}_{\mathrm{w}} \sim 3\left(\frac{2 \mathrm{C}_{\mathrm{f}}}{\mathrm{C}_{\mathrm{D}}}\right)^{2} \mathrm{~L} \rho_{\mathrm{w}} \mathrm{V}_{\mathrm{w}}^{2}
$$

From the balance between Eqs. (1) and (5) we deduce that

$$
\frac{\mathrm{H}}{\mathrm{L}} \sim 3\left(\frac{2 \mathrm{C}_{\mathrm{f}}}{\mathrm{C}_{\mathrm{D}}}\right)^{2} \frac{\rho_{\mathrm{w}}}{\rho_{\mathrm{a}}}\left(\frac{\mathrm{V}_{\mathrm{w}}}{\mathrm{V}_{\mathrm{a}}}\right)^{2}
$$

which in view of $\mathrm{C}_{\mathrm{D}} \sim 1, \mathrm{C}_{\mathrm{f}} \sim 10^{-2}$, and $\rho_{\mathrm{w}} / \rho_{\mathrm{a}} \sim 10^{3}$, becomes

$$
\frac{\mathrm{V}_{\mathrm{w}}}{\mathrm{V}_{\mathrm{a}}} \sim\left(\frac{\mathrm{H}}{\mathrm{L}}\right)^{1 / 2}
$$

In the evolutionary pursuit of higher boat speeds $V_{w}$, the height of the sail approaches the length of the hull. Expressed in terms of scale analysis, the conclusion is that $\mathrm{H}$ and $\mathrm{L}$ must have the same scale because $\mathrm{V}_{\mathrm{w}}$ and $\mathrm{V}_{\mathrm{a}}$ represent the same scale (no wind, no travel; fast wind, fast travel).

With the three aspect ratios now predicted $\left(D_{x} / D_{y}, L / D_{x}, H / L\right)$ the evolutionary direction of the boat model selected in Fig. 1 is complete, and can be drawn in three dimensions. The shape viewed from above is $L / D_{x}$, while from the front and from the side it is respectively $D_{x} / D_{y}$ and $H / L$. There are only three shapes because the configuration of the simplest model (Fig. 1) has only three degrees of freedom. Each of these shapes refers to the external look of the model.

Boat designs are more complicated because in addition to external shape they also have internal structure. The internal structure has additional geometric details, which have increased in number during boat evolution. Three frames from this evolutionary sequence are aligned chronologically in Fig. 3, from two thousand years ago (Egyptian galley) to Columbus crossing the Atlantic (1492) and Napoleon's navy (1800). In antiquity the internal structure was the simplest: one mast supporting one sail. Over time, the sails and the masts became more numerous as the speed and carrying capacity of the boats increased for the benefit of the people who constructed, owned and operated them. Modern monohulled sailboats align more with the Egyptian galley in that there is typically a single mast. The fastest monohulls in the world have a single mast and achieve maximum speeds with $2-3$ sails.

In Fig. 3 the three boats are presented in frames of the same size in order to stress two additional points. In time, the complexity of the architecture increases as the internal structure morphs. Yet, in every frame the external shapes are the same as those that we predicted for the simplest model without internal structure (Fig. 1).

Features of internal structure can be predicted by continuing the analysis started in Eqs. (1-7). Assume that the lone sail in Fig. 1 is supported by one mast, which is modeled as an elastic rod of height $\mathrm{H}$ and diameter $\mathrm{d}$. The mast bends under the horizontal force received from the sail, which is $\mathrm{F}_{\mathrm{a}}$, Eq. (1). The mast is a beam in pure bending, because it is slender enough so that its slenderness $\mathrm{H} / \mathrm{d}$ exceeds 50 .

The highest stresses occur at the base of the mast, where the bending moment is maximum and of order $\mathrm{F}_{\mathrm{a}} \mathrm{H}$. This moment is balanced by the moment due to the nonuniform distribution of stresses in the mast cross section. The stresses are tensile on the forward (convex) side of the mast in bending, and compressive on the aft (concave) side. If the material is such that $\sigma$ is the order of magnitude of the highest allowable (tensile and compressive) stresses at the base, then the bending couple in the cross section is of order $\sigma \mathrm{d}^{2} \times \mathrm{d}$, where the $\sigma \mathrm{d}^{2}$ are the forces 

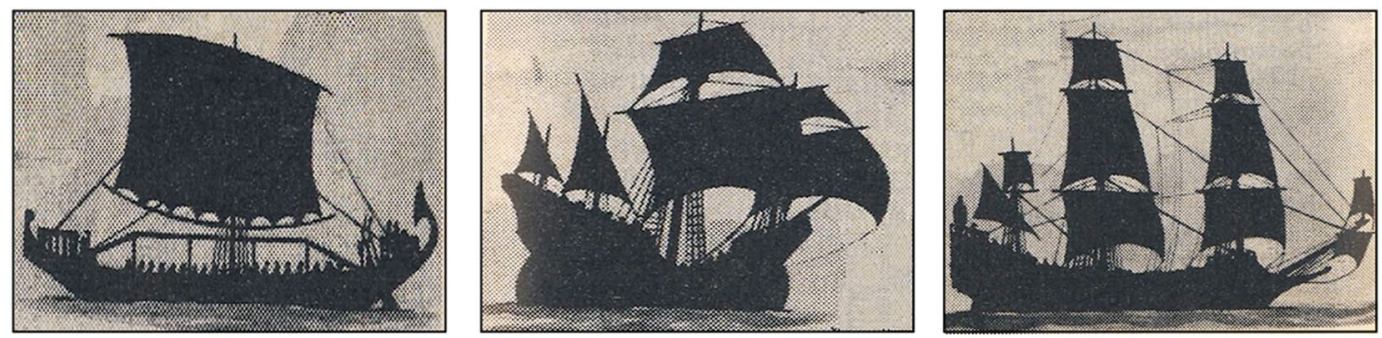

Figure 3. The evolution of boats with sails over the past two thousand years: Egyptian galley, Columbus and Napoleon.

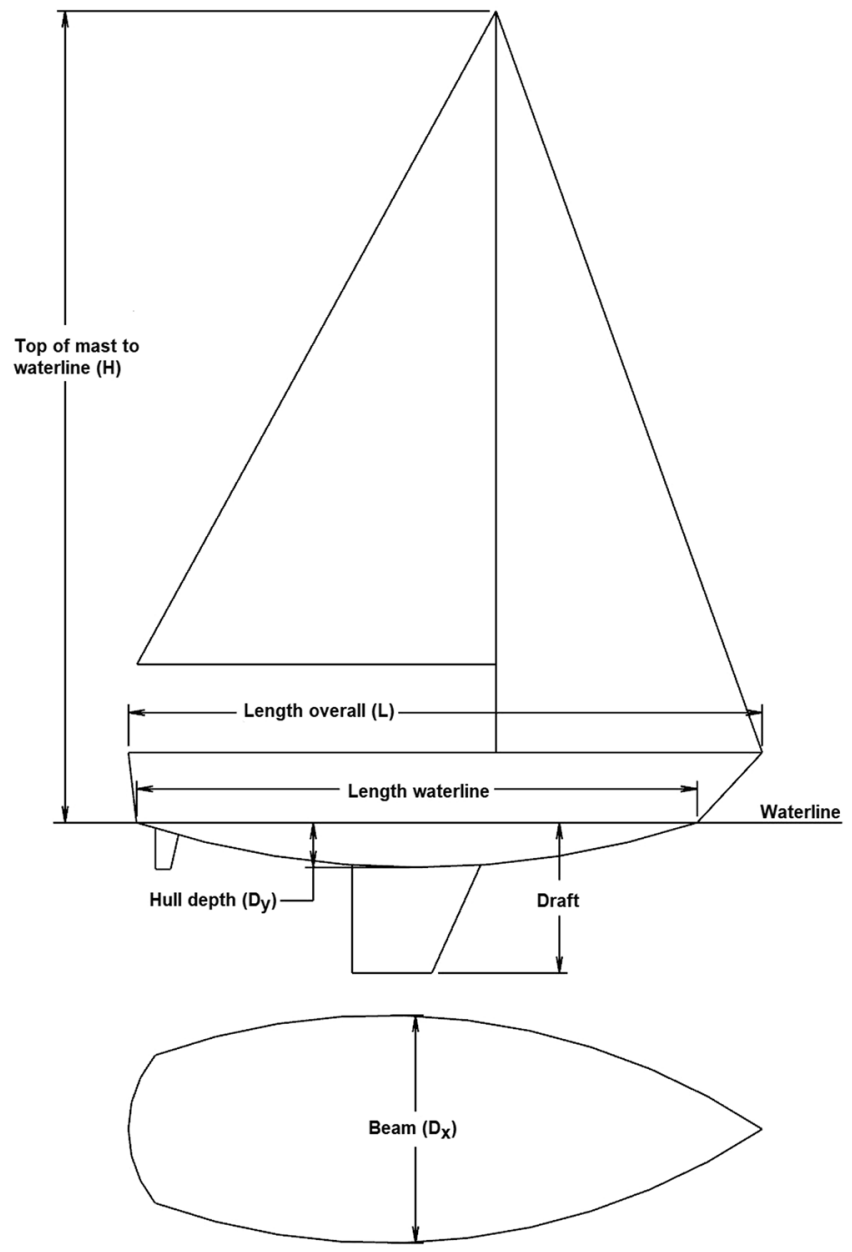

Figure 4. The main dimensions used in yacht construction.

of the couple (tensile and compressive, both aligned with the mast) and dis the arm of the couple, which is transversal to the mast. From the requirement of rotational equilibrium, $\mathrm{F}_{\mathrm{a}} \mathrm{H} \sim \sigma \mathrm{d}^{3}$, we obtain

$$
\frac{\mathrm{d}^{3}}{\mathrm{H}^{2} \mathrm{~L}} \sim \frac{\rho \mathrm{a} \mathrm{V}_{\mathrm{a}}^{2}}{\sigma}
$$

which, in view of Eq. (7), becomes

$$
\frac{\mathrm{d}}{\mathrm{H}} \sim\left(\frac{\rho \mathrm{a} \mathrm{V}_{\mathrm{a}}^{2}}{\sigma}\right)^{1 / 3}\left(\frac{\mathrm{V}_{\mathrm{a}}}{\mathrm{V}_{\mathrm{w}}}\right)^{2 / 3}
$$




\begin{tabular}{|c|c|c|c|c|c|c|c|c|c|c|c|c|c|}
\hline \multirow[b]{2}{*}{ Model } & \multirow[b]{2}{*}{ Year } & \multirow{2}{*}{\begin{tabular}{|l}
$\begin{array}{l}\text { Length } \\
\text { Overall } \\
\text { (L) }\end{array}$ \\
$(\mathrm{m})$ \\
\end{tabular}} & \multirow{2}{*}{\begin{tabular}{|l}
$\begin{array}{l}\text { Length } \\
\text { Waterline (L) }\end{array}$ \\
(m)
\end{tabular}} & \multirow{2}{*}{\begin{tabular}{|l}
$\begin{array}{l}\text { Beam } \\
(\mathbf{D x})\end{array}$ \\
$(\mathbf{m})$ \\
\end{tabular}} & \multirow{2}{*}{\begin{tabular}{|l}
$\begin{array}{l}\text { Sail Area } \\
(\mathbf{H L} / \mathbf{2})\end{array}$ \\
$(\mathrm{m} 2)$ \\
\end{tabular}} & \multirow{2}{*}{ 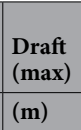 } & \multirow{2}{*}{$\begin{array}{l}\text { Displacement } \\
\mathrm{kg}\end{array}$} & \multirow{2}{*}{\begin{tabular}{|l|} 
Ballast \\
$\mathbf{k g}$
\end{tabular}} & \multirow{2}{*}{\begin{tabular}{|l} 
PHRF \\
Rating
\end{tabular}} & \multirow{2}{*}{\begin{tabular}{|l}
$\begin{array}{l}\text { Hull } \\
\text { Depth } \\
\text { (Dy) }\end{array}$ \\
$(\mathbf{m})$ \\
\end{tabular}} & \multirow{2}{*}{$\begin{array}{l}\text { Height } \\
\text { (H) }\end{array}$} & \multirow[b]{2}{*}{ Hull Shape } & \multirow[b]{2}{*}{ Rig Setup } \\
\hline & & & & & & & & & & & & & \\
\hline Aerodyne 47 & 2001 & 14.2 & 12.83 & 4.37 & 87.14 & 1.83 & 11508 & 4686 & 42 & 0.6 & 18.43 & $\begin{array}{l}\text { Fin w/bulb \& } \\
\text { spade rudder }\end{array}$ & Fractional Sloop \\
\hline Alajuela 33 & 1977 & 10.06 & 8.38 & 3.25 & 53.42 & 1.45 & 6124 & 2132 & 198 & 0.82 & 13.4 & $\begin{array}{l}\text { Fin w/rudder } \\
\text { on skeg }\end{array}$ & Cutter \\
\hline Alberg 35 & 1961 & 10.59 & 7.32 & 2.95 & 50.63 & 1.57 & 5715 & 2404 & 201 & 0.57 & 13.04 & Long keel & Masthead Sloop \\
\hline Alerion & 1996 & 6.1 & 5.21 & 2.08 & 20.44 & 1.07 & 862 & 354 & 249 & 0.25 & 9.74 & $\begin{array}{l}\text { Fin w/bulb \& } \\
\text { spade rudder }\end{array}$ & Fractional Sloop \\
\hline Archabault 31 & 2009 & 9.55 & 8.3 & 3.23 & 50.91 & 1.9 & 3150 & 1350 & 111 & 0.47 & 13.94 & $\begin{array}{l}\text { Fin w/spade } \\
\text { rudder }\end{array}$ & Fractional Sloop \\
\hline B-25 & 1989 & 7.62 & 6.48 & 2.54 & 25.36 & 1.52 & 907 & 363 & 141 & 0.29 & 11.05 & Lifting keel & Fractional Sloop \\
\hline Baba 40 & 1980 & 12.14 & 10.52 & 3.91 & 80.27 & 1.83 & 13154 & 5445 & 183 & 0.63 & 16.85 & Long keel & Cutter \\
\hline Balboa 26 & 1969 & 7.8 & 6.35 & 2.44 & 27.5 & 1.52 & 1633 & 544 & 225 & 0.37 & 10.32 & Swing keel & Masthead Sloop \\
\hline Baltic 37 & 1978 & 11.28 & 8.31 & 3.66 & 59.36 & 2.03 & 6169 & 2776 & 114 & 0.57 & 15.48 & $\begin{array}{l}\text { Fin w/spade } \\
\text { rudder }\end{array}$ & Masthead Sloop \\
\hline Baltic 51 & 1984 & 16.76 & 14.44 & 4.92 & 136.1 & 2.19 & 17500 & 7321 & 51 & 1.08 & 22.3 & $\begin{array}{l}\text { Fin w/spade } \\
\text { rudder }\end{array}$ & Masthead Sloop \\
\hline Beneteau 49 & 2005 & 15.09 & 13.31 & 4.5 & 95.13 & 1.75 & 12935 & 4300 & 54 & 0.74 & 18.38 & $\begin{array}{l}\text { Fin w/bulb \& } \\
\text { spade rudder }\end{array}$ & Fractional Sloop \\
\hline Bianca 111 & 1975 & 11.13 & 9.4 & 3.2 & 68.28 & 1.98 & 6087 & 2794 & 93 & 0.62 & 16.07 & $\begin{array}{l}\text { Fin w/spade } \\
\text { rudder }\end{array}$ & Fractional Sloop \\
\hline Black Watch 37 & 1965 & 11.28 & 7.62 & 3.2 & 55.46 & 1.55 & 7031 & 1905 & 183 & 0.7 & 14.95 & Fin keel & Masthead Yawl \\
\hline $\begin{array}{l}\text { Boothbay Harbor } \\
\text { OD }\end{array}$ & 1938 & 6.4 & 6.02 & 1.68 & 21.09 & 1.07 & 953 & 408 & 252 & 0.29 & 8.49 & Fin keel & Fractional Sloop \\
\hline $\begin{array}{l}\text { Bravura Sportster } \\
29\end{array}$ & 2001 & 9.12 & 7.87 & 2.9 & 46.08 & 2.03 & 1814 & 816 & 72 & 0.32 & 14.5 & Fin keel & Fractional Sloop \\
\hline Brewer 12.8 & 1983 & 12.8 & 10.29 & 4.11 & 82.4 & 2.74 & 10818 & 4990 & 126 & 0.77 & 16.93 & Keel/CB & Cutter \\
\hline Bridges Point 24 & 1985 & 7.32 & 5.69 & 2.36 & 35.12 & 1.04 & 1789 & 1270 & 243 & 0.67 & 9.84 & Long keel & Fractional Sloop \\
\hline Bristol 47.7 & 1979 & 14.33 & 11.35 & 4.01 & 89.93 & 3.35 & 15722 & 6804 & 114 & 0.77 & 18.27 & Keel/Cbrd. & Masthead \\
\hline Buccaneer 220 & 1978 & 6.86 & 5.87 & 2.41 & 19.04 & 0.91 & 1111 & 431 & 234 & 0.29 & 9.37 & $\begin{array}{l}\text { Fin w/trans. } \\
\text { hung rudder }\end{array}$ & Fractional Sloop \\
\hline Bullseye & 1914 & 4.79 & 3.82 & 1.78 & 13.01 & 1.65 & 612 & 340 & 360 & 0.56 & 6.9 & $\begin{array}{l}\text { Long keel w/ } \\
\text { trans. hung } \\
\text { rudder }\end{array}$ & Fractional Sloop \\
\hline Buzzard's Bay 15 & 1899 & 7.47 & 4.57 & 2.06 & 30.75 & 1.68 & 1103 & 454 & 219 & 0.37 & 8.2 & Keel/Cbrd. & Gaffhead \\
\hline C\&C 115 & 2005 & 11.51 & 10.06 & 3.63 & 72.55 & 2.03 & 5352 & 1905 & 66 & 0.52 & 16.9 & $\begin{array}{l}\text { Fin w/bulb \& } \\
\text { spade rudder }\end{array}$ & Fractional Sloop \\
\hline Cal 24 (Hunt) & 1983 & 7.52 & 6.1 & 1.3 & 24.25 & 1.3 & 1497 & 533 & 219 & 0.32 & 9.56 & $\begin{array}{l}\text { Fin w/spade } \\
\text { rudder }\end{array}$ & Masthead Sloop \\
\hline Cambria 44 & 1985 & 14 & 11.07 & 4.11 & 88.53 & 1.8 & 12973 & 5216 & 87 & 0.7 & 19.2 & $\begin{array}{l}\text { Fin w/rudder } \\
\text { on skeg }\end{array}$ & Cutter \\
\hline Cape Dory 25 & 1973 & 7.57 & 5.49 & 2.21 & 24.34 & 0.91 & 1814 & 771 & 261 & 0.5 & 9.33 & Long keel & Masthead Sloop \\
\hline Carrera 290 & 1992 & 8.89 & 8.21 & 2.88 & 38.93 & 1.7 & 1338 & 603 & 99 & 0.14 & 14.28 & $\begin{array}{l}\text { Fin w/bulb \& } \\
\text { spade rudder }\end{array}$ & Fractional Sloop \\
\hline Cartwright 40 & 1975 & 12.19 & 9.35 & 3.43 & 73.3 & 1.78 & 10660 & 4763 & 153 & 1.19 & 15.31 & Long keel & Cutter \\
\hline D\&M 22 & 1971 & 6.71 & 5.72 & 2.57 & 23.6 & 1.68 & 1259 & 454 & 258 & 0.36 & 9.71 & $\begin{array}{l}\text { Fin w/trans. } \\
\text { hung rudder }\end{array}$ & Masthead Sloop \\
\hline Dehler 39 & 1996 & 11.89 & 10.69 & 3.82 & 95.87 & 1.95 & 7001 & 2945 & 81 & 0.45 & 17.16 & $\begin{array}{l}\text { Fin w/bulb \& } \\
\text { spade rudder }\end{array}$ & Fractional Sloop \\
\hline DK 46 & 2002 & 14.1 & 12.35 & 4.1 & 134.89 & 3 & 8650 & 4300 & -30 & 0.54 & 22.95 & $\begin{array}{l}\text { Fin w/bulb \& } \\
\text { spade rudder }\end{array}$ & Fractional Sloop \\
\hline Dolphin $24(\mathrm{~S} \& S)$ & 1959 & 7.36 & 5.79 & 2.34 & 27.59 & 1.58 & 1928 & 748 & 246 & 0.46 & 10.12 & Keel/Cbrd. & Masthead Sloop \\
\hline Dufour 2800 & 1977 & 8.25 & 6.75 & 2.93 & 30.19 & 1.46 & 2751 & 900 & 204 & 0.39 & 10.9 & $\begin{array}{l}\text { Fin w/spade } \\
\text { rudder }\end{array}$ & Masthead Sloop \\
\hline Electra (Person) & 1960 & 6.86 & 5.11 & 2.13 & 21.18 & 0.91 & 1361 & 589 & 288 & 0.39 & 8.96 & Fin keel & Masthead Sloop \\
\hline Endeavour 32 & 1976 & 9.75 & 7.7 & 2.97 & 43.11 & 1.28 & 5307 & 2268 & 190 & 0.58 & 13.21 & $\begin{array}{l}\text { Fin w/rudder } \\
\text { on skeg }\end{array}$ & Masthead Sloop \\
\hline Ericson 31-2 & 1967 & 9.22 & 7.11 & 2.9 & 38.09 & 1.47 & 3538 & 1361 & 168 & 0.46 & 13.29 & $\begin{array}{l}\text { Fin w/spade } \\
\text { rudder }\end{array}$ & Masthead Sloop \\
\hline Esprit 37 & 1977 & 11.28 & 9.63 & 3.51 & 61.96 & 1.77 & 7711 & 3039 & 150 & 0.7 & 17.37 & $\begin{array}{l}\text { Fin w/rudder } \\
\text { on skeg }\end{array}$ & Cutter \\
\hline Etchelles ODR & 1966 & 9.3 & 6.71 & 2.13 & 27.96 & 1.37 & 1508 & 987 & 120 & 0.4 & 11.71 & $\begin{array}{l}\text { Fin w/rudder } \\
\text { on skeg }\end{array}$ & Fractional Sloop \\
\hline Express 37 & 1984 & 11.3 & 9.4 & 3.51 & 59.36 & 2.21 & 4445 & 2087 & 72 & 0.43 & 15.43 & $\begin{array}{l}\text { Fin w/spade } \\
\text { rudder }\end{array}$ & Masthead Sloop \\
\hline Farr 36 OD & 2002 & 11 & 10 & 3.57 & 51.28 & 2.59 & 3059 & 1596 & 0 & 0.28 & 17.81 & Lifting keel & Fractional Sloop \\
\hline Continued & & & & & & & & & & & & & \\
\hline
\end{tabular}




\begin{tabular}{|c|c|c|c|c|c|c|c|c|c|c|c|c|c|}
\hline \multirow[b]{2}{*}{ Model } & \multirow[b]{2}{*}{ Year } & \multirow{2}{*}{\begin{tabular}{|l}
$\begin{array}{l}\text { Length } \\
\text { Overall } \\
\text { (L) }\end{array}$ \\
$(\mathrm{m})$
\end{tabular}} & \multirow{2}{*}{\begin{tabular}{|l} 
Length \\
Waterline (L) \\
(m)
\end{tabular}} & \multirow{2}{*}{ 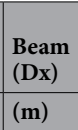 } & \multirow{2}{*}{\begin{tabular}{|l} 
Sail Area \\
$(\mathrm{HL} / 2)$
\end{tabular}} & \multirow{2}{*}{\begin{tabular}{|l}
$\begin{array}{l}\text { Draft } \\
(\mathbf{m a x})\end{array}$ \\
$(\mathbf{m})$ \\
\end{tabular}} & \multirow{2}{*}{\begin{tabular}{|l|} 
Displacement \\
$\mathrm{kg}$
\end{tabular}} & \multirow{2}{*}{\begin{tabular}{|l|} 
Ballast \\
$\mathbf{k g}$
\end{tabular}} & \multirow{2}{*}{\begin{tabular}{|l} 
PHRF \\
Rating
\end{tabular}} & \multirow{2}{*}{\begin{tabular}{|l|}
$\begin{array}{l}\text { Hull } \\
\text { Depth } \\
\text { (Dy) }\end{array}$ \\
$(\mathrm{m})$ \\
\end{tabular}} & \multirow{2}{*}{$\begin{array}{l}\text { Height } \\
\text { (H) }\end{array}$} & \multirow[b]{2}{*}{ Hull Shape } & \multirow[b]{2}{*}{ Rig Setup } \\
\hline & & & & & & & & & & & & & \\
\hline Fast Passage 39 & 1976 & 12.04 & 10.21 & 3.61 & 74.23 & 1.68 & 9526 & 3402 & 132 & 0.77 & 16.82 & $\begin{array}{l}\text { Fin w/rudder } \\
\text { on skeg }\end{array}$ & Cutter \\
\hline Figaro Solo & 1989 & 9.14 & 8.4 & 3.25 & 48.77 & 1.8 & 2400 & 900 & 48 & 0.34 & 13.22 & $\begin{array}{l}\text { Fin w/bulb \& } \\
\text { spade rudder }\end{array}$ & Fractional Sloop \\
\hline Finngulf 37 & 2004 & 11.25 & 10 & 3.5 & 64.64 & 2 & 6500 & 2250 & 84 & 0.48 & 16.85 & $\begin{array}{l}\text { Fin keel w/ } \\
\text { bulb }\end{array}$ & Fractional Sloop \\
\hline Flying Tiger $10 \mathrm{~m}$ & 2005 & 9.95 & 9.24 & 2.79 & 49.89 & 2.32 & 1984 & 870 & 54 & 0.32 & 14.61 & Lifting keel & Fractional Sloop \\
\hline Gladiator 24 & 1958 & 7.32 & 6.1 & 2.29 & 25.73 & 1.22 & 1746 & 930 & 258 & 0.48 & 10.83 & Fin keel & Fractional Sloop \\
\hline Grampian 30 & 1969 & 9.07 & 7.77 & 2.9 & 39.48 & 1.42 & 3901 & 1755 & 192 & 0.73 & 13.38 & $\begin{array}{l}\text { Fin w/spade } \\
\text { rudder }\end{array}$ & Masthead Sloop \\
\hline Hallburg Rassy 31 & 1992 & 9.56 & 7.74 & 3.32 & 46.17 & 1.73 & 4572 & 2032 & 198 & 0.57 & 13.51 & $\begin{array}{l}\text { Fin w/spade } \\
\text { rudder }\end{array}$ & Fractional Sloop \\
\hline Hanse 385 & 2011 & 11.4 & 10.4 & 3.88 & 67.26 & 1.99 & 7600 & 2200 & 99 & 0.53 & 16.98 & $\begin{array}{l}\text { Fin w/bulb \& } \\
\text { spade rudder }\end{array}$ & Fractional Sloop \\
\hline Harbor 20 & 1997 & 6 & 5.18 & 2.13 & 20.44 & 1.07 & 816 & 408 & 225 & 0.22 & 9.17 & $\begin{array}{l}\text { Fin w/bulb \& } \\
\text { spade rudder }\end{array}$ & Fractional Sloop \\
\hline Harpoon 6.2 & 1979 & 6.2 & 4.82 & 2.44 & 17.56 & 1.12 & 771 & 249 & 240 & 0.25 & 8.33 & $\begin{array}{l}\text { Fin w/trans. } \\
\text { hung rudder }\end{array}$ & Fractional Sloop \\
\hline Henderson 30 & 1997 & 9.4 & 8.76 & 3 & 44.59 & 2.13 & 1746 & 794 & 45 & 0.29 & 15.83 & Lifting keel & Fractional Sloop \\
\hline Hunter 336 & 1995 & 10.21 & 8.71 & 3.56 & 53.23 & 1.37 & 5003 & 1860 & 147 & 0.43 & 17.02 & $\begin{array}{l}\text { Fin w/bulb \& } \\
\text { spade rudder }\end{array}$ & Fractional Sloop \\
\hline Irwin 23 & 1968 & 7.01 & 5.64 & 2.44 & 23.78 & 1.75 & 1452 & 680 & 252 & 0.38 & 9.4 & \begin{tabular}{|l}
$\begin{array}{l}\text { Keel/CB \& } \\
\text { spade rudder }\end{array}$ \\
\end{tabular} & Masthead Sloop \\
\hline Islander 29 & 1968 & 8.86 & 7.11 & 2.69 & 36.42 & 1.12 & 3538 & 1134 & 234 & 0.51 & 11.35 & Fin keel & Masthead Sloop \\
\hline $\begin{array}{l}\text { Islander Packet } \\
320\end{array}$ & 1998 & 10.13 & 8.23 & 3.58 & 51.65 & 1.3 & 6124 & 2722 & 198 & 0.77 & 14.84 & Long keel & Cutter \\
\hline IW-31 & 1968 & 9.24 & 6.71 & 2.69 & 33.17 & 1.65 & 3538 & 1542 & 192 & 0.64 & 10.84 & $\begin{array}{l}\text { Fin w/rudder } \\
\text { on skeg }\end{array}$ & Masthead Sloop \\
\hline$J / 111$ & 2010 & 11.1 & 9.97 & 3.29 & 61.22 & 2.19 & 4350 & 1595 & 42 & 0.39 & 15.86 & $\begin{array}{l}\text { Fin w/bulb \& } \\
\text { spade rudder }\end{array}$ & Fractional Sloop \\
\hline$J / 160$ & 1996 & 16.06 & 14.48 & 4.42 & 127.83 & 2.13 & 14152 & 5443 & -9 & 0.7 & 22.1 & $\begin{array}{l}\text { Fin w/bulb \& } \\
\text { spade rudder }\end{array}$ & Fractional Sloop \\
\hline Jonmeri 48 & 1988 & 14.52 & 12.2 & 4.74 & 109.16 & 2.51 & 15640 & 7100 & 75 & 0.81 & 20.2 & $\begin{array}{l}\text { Fin w/spade } \\
\text { rudder }\end{array}$ & Masthead Sloop \\
\hline Kirby 25 & 1974 & 7.67 & 6.32 & 2.67 & 26.85 & 1.27 & 1429 & 522 & 177 & 0.2 & 10.68 & $\begin{array}{l}\text { Fin w/spade } \\
\text { rudder }\end{array}$ & Fractional Sloop \\
\hline Knutson35 & 1955 & 10.67 & 7.62 & 3 & 53.05 & 1.47 & 7711 & 2431 & 195 & 0.83 & 14.7 & Fin keel & Fractional Sloop \\
\hline Lafitte 44 & 1978 & 13.51 & 10.82 & 3.86 & 89.83 & 1.93 & 12701 & 5130 & 126 & 0.77 & 17.4 & $\begin{array}{l}\text { Fin w/rudder } \\
\text { on skeg }\end{array}$ & Cutter \\
\hline Lager 40 & 1984 & 12.09 & 10.13 & 3.86 & 74.41 & 2.18 & 5579 & 3039 & 60 & 0.58 & 17.05 & $\begin{array}{l}\text { Fin } w / \text { spade } \\
\text { rudder }\end{array}$ & Fractional Sloop \\
\hline Little Harbor 60 & 1995 & 18.42 & 14.55 & 4.93 & 139.81 & 3.63 & 31979 & 10433 & 48 & 1.2 & 22.42 & Keel/Cbrd. & Cutter \\
\hline Luders 16 & 1933 & 8.03 & 4.98 & 1.75 & 20.81 & 1.22 & 1338 & 726 & 216 & 0.45 & 9.96 & Long keel & Masthead Yawl \\
\hline Medalist 33 & 1965 & 10.06 & 7.34 & 3.05 & 42.36 & 1.6 & 5307 & 1905 & 234 & 0.87 & 13.89 & $\begin{array}{l}\text { Fin w/spade } \\
\text { rudder }\end{array}$ & Masthead Sloop \\
\hline Mistress 32 & 1969 & 9.7 & 6.9 & 2.84 & 41.34 & 1.85 & 3400 & 1500 & 195 & 0.63 & 11.25 & $\begin{array}{l}\text { Fin w/rudder } \\
\text { on skeg }\end{array}$ & Masthead Sloop \\
\hline Monhegan 48 & 1998 & 14.82 & 11.43 & 4.19 & 96.71 & 1.52 & 12746 & 5443 & 72 & 0.76 & 19.09 & Keel/Cbrd. & Masthead Sloop \\
\hline Moody 45 DS & 2010 & 13.72 & 12.93 & 4.57 & 97.92 & 1.99 & 13600 & 4300 & 108 & 0.81 & 20.52 & $\begin{array}{l}\text { Fin w/bulb \& } \\
\text { twin rudders }\end{array}$ & Fractional Sloop \\
\hline Mumm 36 & 1993 & 10.92 & 9.68 & 3.61 & 59.83 & 2.23 & 3697 & 1588 & 42 & 0.38 & 16.8 & $\begin{array}{l}\text { Fin w/bulb \& } \\
\text { spade rudder }\end{array}$ & Fractional Sloop \\
\hline Najad 373 & 1999 & 11.3 & 9.75 & 3.65 & 59.46 & 1.9 & 8300 & 3100 & 156 & 0.64 & 17.1 & $\begin{array}{l}\text { Fin w/rudder } \\
\text { on skeg }\end{array}$ & Masthead Sloop \\
\hline Nevins 40 & 1955 & 12.19 & 8.38 & 3.43 & 68.65 & 2.31 & 9979 & 2313 & 168 & 0.83 & 14.33 & Keel/Cbrd. & Masthead Yawl \\
\hline Newport 28 & 1974 & 8.46 & 7.62 & 2.9 & 36.7 & 1.3 & 3175 & 1452 & 195 & 0.58 & 12.64 & $\begin{array}{l}\text { Fin w/spade } \\
\text { rudder }\end{array}$ & Masthead Sloop \\
\hline Nordic 44 & 1980 & 13.36 & 10.8 & 3.94 & 85.38 & 2.13 & 10546 & 4237 & 84 & 0.65 & 18.78 & $\begin{array}{l}\text { Fin w/rudder } \\
\text { on skeg }\end{array}$ & Masthead Sloop \\
\hline Ohlson 36 & 1958 & 10.97 & 7.62 & 2.84 & $\mathrm{n} / \mathrm{a}$ & 1.85 & 6260 & 2268 & 189 & 0.86 & 12.56 & Fin keel & Masthead Sloop \\
\hline Oyster 485 & 1994 & 14.78 & 11.43 & 4.27 & 89.18 & 2.13 & 17033 & 590 & 84 & 0.84 & 18.08 & $\begin{array}{l}\text { Fin w/rudder } \\
\text { on skeg }\end{array}$ & Cutter \\
\hline Puma 23 & 1971 & 6.87 & 5.39 & 2.21 & 22.76 & 1.18 & 1347 & 675 & 258 & 0.43 & 9.77 & $\begin{array}{l}\text { Fin w/rudder } \\
\text { on skeg }\end{array}$ & Masthead Sloop \\
\hline Quickstep 24 & 1976 & 7.29 & 5.79 & 2.41 & 24.06 & 1.04 & 1814 & 862 & 240 & 0.36 & 9.39 & $\begin{array}{l}\text { Fin w/rudder } \\
\text { on skeg }\end{array}$ & Masthead Sloop \\
\hline
\end{tabular}




\begin{tabular}{|c|c|c|c|c|c|c|c|c|c|c|c|c|c|}
\hline \multirow[b]{2}{*}{ Model } & \multirow[b]{2}{*}{ Year } & \multirow{2}{*}{\begin{tabular}{|l|}
$\begin{array}{l}\text { Length } \\
\text { Overall } \\
\text { (L) }\end{array}$ \\
$(\mathrm{m})$ \\
\end{tabular}} & \multirow{2}{*}{\begin{tabular}{|l} 
Length \\
Waterline (L) \\
$(\mathbf{m})$
\end{tabular}} & \multirow{2}{*}{\begin{tabular}{|l|} 
Beam \\
$(\mathbf{D x})$
\end{tabular}} & \multirow{2}{*}{ 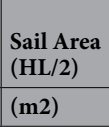 } & \multirow{2}{*}{\begin{tabular}{|l|}
$\begin{array}{l}\text { Draft } \\
(\mathbf{m a x})\end{array}$ \\
$(\mathbf{m})$ \\
\end{tabular}} & \multirow{2}{*}{\begin{tabular}{|l} 
Displacement \\
$\mathrm{kg}$
\end{tabular}} & \multirow{2}{*}{\begin{tabular}{|l|} 
Ballast \\
$\mathrm{kg}$ \\
\end{tabular}} & \multirow{2}{*}{\begin{tabular}{|l|}
$\begin{array}{l}\text { PHRF } \\
\text { Rating }\end{array}$ \\
s/nm \\
\end{tabular}} & \multirow{2}{*}{$\begin{array}{l}\text { Hull } \\
\text { Depth } \\
\text { (Dy) }\end{array}$} & \multirow{2}{*}{$\begin{array}{l}\text { Height } \\
\text { (H) }\end{array}$} & \multirow[b]{2}{*}{ Hull Shape } & \multirow[b]{2}{*}{ Rig Setup } \\
\hline & & & & & & & & & & & & & \\
\hline Ranger 33 & 1969 & 10.11 & 8 & 2.92 & 49.14 & 1.52 & 4763 & 2041 & 156 & 0.45 & 13.39 & $\begin{array}{l}\text { Fin w/spade } \\
\text { rudder }\end{array}$ & Masthead Sloop \\
\hline Redwing 30 & 1967 & 9.23 & 6.63 & 2.68 & 37.53 & 1.37 & 3383 & 1647 & 195 & 0.77 & 10.86 & $\begin{array}{l}\text { Fin w/spade } \\
\text { rudder }\end{array}$ & Masthead Sloop \\
\hline Rhodes 41 & 1961 & 12.44 & 8.53 & 3.12 & 67.35 & 1.75 & 8528 & 3663 & 171 & 0.87 & 14.05 & Long keel & Masthead Sloop \\
\hline Sabre 386 & 2004 & 11.79 & 9.91 & 3.86 & 69.12 & 2.08 & 7688 & 2903 & 84 & 0.6 & 16.45 & $\begin{array}{l}\text { Fin w/bulb \& } \\
\text { spade rudder }\end{array}$ & Masthead Sloop \\
\hline Sabre 426 & 2002 & 12.95 & 10.97 & 4.09 & 85.47 & 2.08 & 10886 & 3810 & 69 & 0.63 & 17.34 & $\begin{array}{l}\text { Fin w/bulb \& } \\
\text { spade rudder }\end{array}$ & Masthead Sloop \\
\hline Saga 43 & 1996 & 13.18 & 11.86 & 3.66 & 88.44 & 1.91 & 9000 & 3538 & 81 & 0.58 & 18.93 & $\begin{array}{l}\text { Fin w/bulb \& } \\
\text { spade rudder }\end{array}$ & Masthead Sloop \\
\hline Sailmaster 22 & 1963 & 6.71 & 5.03 & 2.13 & 21.27 & 1.8 & 1656 & 635 & 294 & 0.33 & 8.96 & Keel/Cbrd. & Masthead Sloop \\
\hline Seafarer 45 & 1961 & 13.74 & 9.24 & 3.35 & 83.42 & 1.96 & 11340 & 4196 & 156 & 1.04 & 15.85 & Fin keel & Masthead Sloop \\
\hline Seascape 27 & 2011 & 7.99 & 7.99 & 2.54 & 45.99 & 1.95 & 1150 & 550 & 105 & 0.26 & 12.25 & $\begin{array}{l}\text { Keel/CB w/ } \\
\text { twin rudders }\end{array}$ & Fractional Sloop \\
\hline Swan 77 & 1992 & 24.01 & 18.38 & 6 & 261.14 & 3.4 & 51001 & 18000 & -12 & 1.41 & 32.11 & $\begin{array}{l}\text { Fin w/spade } \\
\text { rudder }\end{array}$ & Masthead Sloop \\
\hline Tartan 3800 & 1994 & 11.58 & 9.45 & 3.79 & 61.78 & 1.62 & 7258 & 3175 & 114 & 0.64 & 16.44 & $\begin{array}{l}\text { Fin w/bulb \& } \\
\text { spade rudder }\end{array}$ & Masthead Sloop \\
\hline Tayana 55 & 1983 & 16.76 & 14 & 4.9 & 129.97 & 1.98 & 21954 & 7963 & 54 & 0.74 & 20.77 & $\begin{array}{l}\text { Fin w/rudder } \\
\text { on skeg }\end{array}$ & Cutter \\
\hline Thunderbird & 1958 & 7.92 & 6.17 & 2.3 & 28.71 & 1.46 & 1656 & 694 & 213 & 0.33 & 10.97 & $\begin{array}{l}\text { Fin w/spade } \\
\text { rudder }\end{array}$ & Fractional Sloop \\
\hline Ultimate 20 & 1994 & 6.35 & 5.49 & 2.44 & 22.57 & 1.52 & 499 & 204 & 144 & 0.16 & 9.72 & Lifting keel & Fractional Sloop \\
\hline Viper 830 & 1996 & 8.44 & 7.62 & 2.59 & 38.83 & 2.18 & 1134 & 700 & 66 & 0.23 & 12.94 & Lifting keel & Fractional Sloop \\
\hline Whistler 48 & 1982 & 14.53 & 12.27 & 4.24 & 108.6 & 1.78 & 16288 & 6196 & 102 & 0.93 & 19.39 & Sheel keel & Cutter \\
\hline $\mathrm{X}-442$ & 1993 & 13.51 & 11.2 & 4.13 & 88.81 & 2.29 & 9662 & 4300 & 36 & 0.63 & 18.58 & $\begin{array}{l}\text { Fin w/bulb \& } \\
\text { spade rudder }\end{array}$ & Masthead Sloop \\
\hline Yankee 26 & 1974 & 7.92 & 6.3 & 2.64 & 27.87 & 1.45 & 2420 & 974 & 264 & 0.6 & 12.94 & $\begin{array}{l}\text { Fin w/rudder } \\
\text { on skeg }\end{array}$ & Masthead Sloop \\
\hline
\end{tabular}

Table 1. Sailboat model data.

To summarize, the predicted evolutionary design has the three external shapes discussed previously, plus one internal shape, $\mathrm{d} / \mathrm{H}$, the mast slenderness. One formula, Eq. (9), governs the evolution of this technology, past and future. A stronger material (larger $\sigma$ ) makes a more slender (thinner, lighter) mast, which in turn decreases the dead weight of the vessel (and the submerged portion of the hull), reduces hull friction and increases the boat speed.

\section{Comparison with Current Designs}

In the modern era, physics principles have played a guiding role in the improvements that have been made in the design of boats with sails. The icon of the central role of physics in boat design is Euler's entry ${ }^{36}$ in the 1727 contest for the King's prize for the solution to the nautical problem to determine the best way to place the masts on vessels, and the relation between their positions and the number and height of the masts. Since then, fluid dynamics and naval engineering grew as scientific domains, as did naval vessel technology before and after the advent of steam power ${ }^{37-42}$. In this section we compare the current state of sail boat architecture with the design features predicted theoretically.

Table 1 consists of 96 single hulled sailboats with a variety of models, years, dimensions, weights, and designs ${ }^{43}$. The variables $\mathrm{D}_{\mathrm{x}}, \mathrm{D}_{\mathrm{y}}, \mathrm{L}$, and $\mathrm{H}$ correlate to Beam, Hull Depth, Overall Length, and Height, respectively, which are defined in Fig. 4 . The displacement $[\mathrm{kg}]$ is the mass of the boat, which is equal to the mass of the water that is displaced by the boat.

PHRF is the performance indicator, which stands for Performance Handicap Racing Fleet. PHRF is a handicapping standardization that equates the performance of different boats. It is designed to rate the boat design characteristics only, and is impartial to the talent of the skipper and crew. With this in mind, the skipper and crew who sail the best overall race from boat handling and tactical perspective should be awarded as the victors.

Boats are given PHRF ratings based on empirical data including dimensional characteristics, materials, past race finishes, similar boats scaling, and comparisons to other handicap systems. The PHRF system also accounts for three ranges of wind conditions (light, moderate, heavy breeze) by utilizing distinct constants for the ratings formula. Like all handicapping systems, PHRF is imperfect due to the opportunity to inject subjectivisms but is the most-widely accepted handicapping systems in the U.S.

For the scope of this article, it should be known that the lower or negative ratings correlate to faster boats. For instance, the fastest boat in Table 1 is the DK 46 with a PHRF rating of 30, and the slowest is the Bullseye with a rating of 360 . In moderate conditions, the DK 46 should be 1.75 times faster boat-for-boat than the Bullseye when utilizing the correction factor formula. The correction factor is then applied to the overall time, generating 


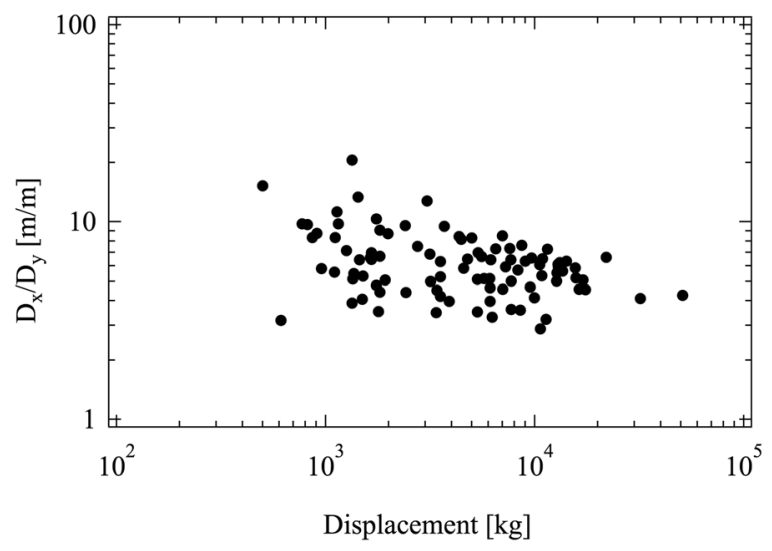

Figure 5. The ratio $D_{x} / D_{y}$ (beam/draft) according to the data presented in Table 1 .

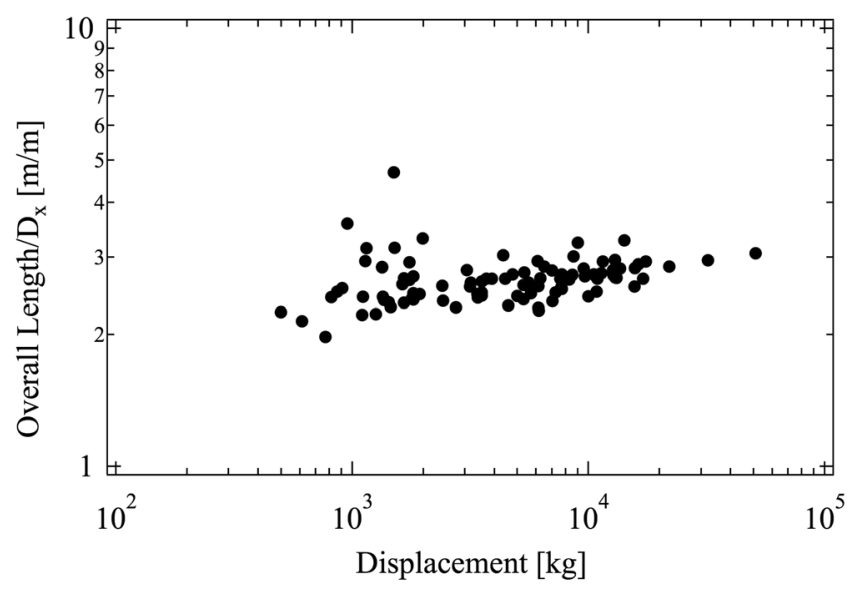

Figure 6. The ratio $\mathrm{L} / \mathrm{D}_{\mathrm{x}}$ (overall length/beam) according to the data presented in Table 1 .

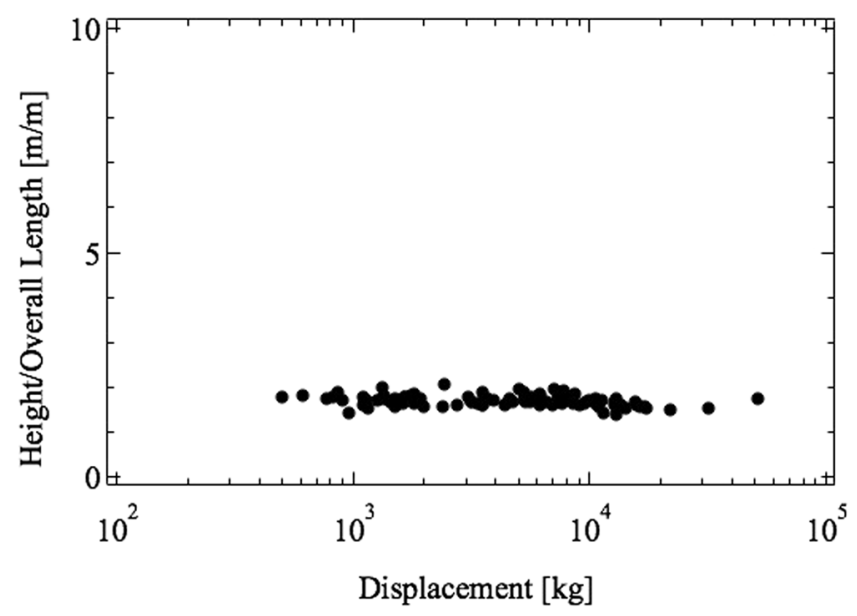

Figure 7. The ratio H/L (height/overall length) according to the data presented in Table 1.

a "corrected time" that generates a one-to-one comparison between the different boats. The boat with the lowest corrected time is the winner of the race.

Plotted in Figs. 5-7 are the actual measurements extracted from Table 1. The three figures show the aspect ratios $D_{x} / D_{y}, L / D_{x}$ and $H / L$ versus the boat displacement. Noteworthy is that the three aspect ratios do not depend 
on the displacement. This means that the main aspects of the configuration did not change over time, as the displacement increased in history (cf. Fig. 3).

The results provided by Eqs. (3), (4) and (7) predict the convergent evolution observed in Figs. 5-7. The geometrical ratios that define the shape of the boats with sails are permanent characteristics over the ages and cultures.

Due to the number of unique designs, calculating the exact performance of a yacht is dependent on a significant number of intrinsic properties as well as external factors. Different boats achieve peak performances at specified degrees from the wind direction in specific conditions. 'Degrees' refers to the boat pointing angle relative to the true wind direction. Ultimately, as the boat moves faster and increases the apparent wind velocity (wind speed plus boat speed), the apparent wind angle becomes small relative to the true wind angle.

The fastest boats in the world are almost always sailing 'upwind' (with sails pulled in closer to the hull mid-line) because they can generate significant boat speed relative to the wind speed. This phenomenon is depicted in Fig. 2. Note the two distinct instances, where the boat speed $\left(\mathrm{S}_{1}<\mathrm{S}_{2}\right)$ and the angle of attack $\left(\beta_{1}>\beta_{2}\right)$ change, while the true wind speed $\mathrm{W}$ is constant. The apparent wind vector $\mathrm{A}$ increases with the boat speed when the boat direction and the true wind vector do not change. The sail's (or airfoil's) leading edge will point in the direction of the apparent wind to generate lift. As the boat speed continues to increase, the apparent wind angle moves forward until the sail is pulled to centerline (cf., Fig. 2), at which point the boat has reached is maximum speed for its relative boat angle from the true wind direction.

There are various calculations to generate boat speed using a characteristic dimension. One example is the critical velocity or $\mathrm{V}_{c} \cong 1.25(\mathrm{LWL})^{1 / 2}$. This formula follows from the fact that the bow of a sailboat moving through the water produces a transverse wave, and at critical velocity the wave extends the length of the waterline, which essential traps the boat in the trough, not allowing it to escape the transverse wave, and so capping its velocity. There are various circumstances that can allow a boat to go faster than $V_{c}$, such as "surfing" external waves or utilizing hydro-foils to lift the hull out of the water.

Received: 2 October 2019; Accepted: 17 January 2020;

Published online: 17 February 2020

\section{References}

1. Bejan, A. Freedom and Evolution: Hierarchy in Nature, Society and Science (Springer Nature, Switzerland AG, 2020).

2. Kakac, S. Evolution of the science of thermodynamics: the history. J. Thermal Science and Technology 36(2), 1-6 (2016).

3. Bejan, A. \& Lorente, S. The constructal law and the evolution of design in nature. Phys. Life Rev. 8, 209-240 (2011).

4. Basak, T. The law of life: The bridge between Physics and Biology. Phys. Life Rev. 8, 249-252 (2011).

5. Delgado, J. M. P. Q. The constructal law: from man-made flow systems to pedestrian flows. Phys. Life Rev. 10, 197-198 (2013).

6. Reis, A. H. Constructal theory: From engineering to physics, and how flow systems develop shape and structure. Appl. Mech. Rev. 59, 269-282 (2006).

7. Chen, L. Progress in the study on constructal theory and its applications. Sci. China, Ser. E: Technol. Sci. 55(3), 802-820 (2012).

8. Reis, A. R. Design in nature, and the laws of physics. Phys. Life Rev. 8, 255-256 (2011).

9. Kasimova, R. Optimal shape of anthill dome: Bejan's constructal law revisited. Ecological Modelling 250, 384-390 (2013).

10. Wang, L. Universality of design and its evolution. Phys. Life Rev. 8, 257-258 (2011).

11. Ventikos, Y. The importance of the constructal framework in understanding and eventually replicating structure in tissue. Phys. Life Rev. 8, 241-242 (2011).

12. Lucia, U., Ponzetto, A. \& Deisbroeck, T. S. A thermo-physical analysis of the proton pump vacuolar-ATPase: the constructal approach. Nature Scientific Reports 4, 6763 (2014).

13. Miguel, A. The emergence of design in pedestrian dynamics: locomotion, self-organization, walking paths and constructal law. Phys. Life Rev. 10, 168-190 (2013).

14. Kasimova, R. G., Tishin, D. \& Kacimov, A. R. Streets and pedestrian trajectories in an urban district: Bejan's constructal principle revisited. Physica A 410, 601-608 (2014).

15. Mavromatidis, L. E., Mavromatidi, A. \& Lequay, H. The unbearable lightness of expertness or space creation in the "climate change" era: a theoretical extension of the "constructal law" for building and urban design. City Cult. Soc. 5, 21-29 (2014).

16. Miguel, A. F. Natural flow systems: acquiring their constructal morphology. Int. J. Design Nature \& Ecodynamics 5, 230-241, https:// doi.org/10.2495/DNE-V5-N3-230-241 (2010).

17. Kacimov, A. R., Klammler, H., Il'yinskii, H. \& Hatfield, K. Constructal design of permeable reactive barriers: groundwater hydraulics criteria. J. Eng. Math. 71, 319-338 (2011).

18. Reis, A. H. \& Gama, C. Sand size versus beachface slope-an explanation based on the constructal law. Geomorphology 114(3), 276-283 (2010).

19. Miguel, A. F. The physics principle of the generation of flow configuration. Physics of Life Reviews 8, 243-244, https://doi. org/10.1016/j.plrev.2011.05.010 (2011).

20. Miguel, A. F. \& Rocha, L. A. O. Tree-shaped Fluid Flow and Heat Transfer. Cham, Switzerland: Springer; 2018.

21. Lorenzini, G., Moretti, S. \& Conti, A. Fin Shape Optimization Using Bejan's Constructal Theory (Morgan \& Claypool Publishers, 2011).

22. Rocha, L. Convection in Channels and Porous Media: Analysis, Optimization, and Constructal Design (VDM Verlag, Saarbrücken, 2009).

23. Lorenzini, G. \& Biserni, C. The Constructal law: From design in nature to social dynamics and wealth as physics. Phys. Life Rev. 8, 259-260 (2011).

24. Reis, A. H., Miguel, A. F. \& Aidin, M. Constructal theory of flow architecture of the lungs. Med. Phys. 31, 1135-1140 (2004).

25. Miguel, A. F. Constructal pattern formation in stony corals, bacterial colonies and plant roots under different hydrodynamics conditions. J. Theor. Biol. 242, 954-961 (2006).

26. Bejan, A. Why the bigger live longer and travel farther: animals, vehicles, rivers and the winds. Nature Scientific Reports 2, 594, https://doi.org/10.1038/srep00594(2012).

27. Bejan, A., Ziaei, S. \& Lorente, S. Evolution: Why all plumes and jets evolve to round cross sections. Nature Scientific Reports 4, 4730, https://doi.org/10.1038/srep04730 (2014).

28. Bejan, A., Lorente, S., Yilbas, B. S. \& Sahin, A. Z. Why solidification has an S-shaped history. Nature Scientific Reports 3, 1711, https:// doi.org/10.1038/srep01711 (2013).

29. Bejan, A. Maxwell's demons everywhere: evolving design as the arrow of time. Nature Scientific Reports 4, 4017, https://doi. org/10.1038/srep04017 (2014). 
30. Bejan, A. \& Périn, S. Constructal theory of Egyptian Pyramids and flow fossils in general, section 13.6 in A. Bejan, Advanced Engineering Thermodynamics, $3^{\text {rd }}$., Wiley, Hoboken, 2013.

31. Bejan, A. Why humans build fires shaped the same way. Nature Scientific Reports 5, 11270, https://doi.org/10.1038/srep11270 (2015).

32. Bejan, A., Charles, J. D. \& Lorente, S. The evolution of airplanes. J. Appl. Phys. 116, 044901 (2014).

33. Chen, R., Wen, C. Y., Lorente, S. \& Bejan, A., The evolution of helicopters. J. Appl. Physics, 120, article 014901 (2016).

34. Bejan, A. The Physics of Life: The Evolution of Everything (St. Martin's Press, New York, 2016).

35. Fossati, F. Aero-Hydrodynamics and the Performance of Sailing Yachts, International Marine/McGraw-Hill First Edition, Camden, Me (2009).

36. Leonhard Euler's 1728 Essay to the French Royal Academy: E004, translated \& annotated by Ian Bruce from Meditationes super problemate nautico de implantatione malorum, quce proxime accessere. Ad premium anno 1727 à Regia Scientiarum Academia promulgatum, Paris, 1728.

37. Daniel Bernoulli, Hydrodynamics, translated by T. Carmody and H. Kobus (Dover, New York, 1968).

38. Johann Bernoulli, Hydraulics, translated by T. Carmody and H. Kobus (Dover, New York, 1968).

39. Lazare Carnot, Essai sur les Machines en Général (Dijon, 1783).

40. Gillispie, C. C. Lazare Carnot, Savant (Princeton University Press, Princeton, NJ, 1971).

41. Prandtl, L. Essentials of Fluid Dynamics (Blackie \& Son, London, 1952).

42. Cardwell, D. S. L. From Watt to Clausius (Cornell University Press, Ithaca, NY, 1971).

43. Sailboat Data Compare Boats, https://sailboatdata.com/sailboat, Accessed: July 2016.

\section{Author contributions}

A.B. and S.L. developed the theory, wrote the manuscript, and made Figures 1 and 3; L.F. collected the boats data (Table 1), contributed to the manuscript, and drew Figures 2 and 4-7.

\section{Competing interests}

The authors declare no competing interests.

\section{Additional information}

Correspondence and requests for materials should be addressed to A.B.

Reprints and permissions information is available at www.nature.com/reprints.

Publisher's note Springer Nature remains neutral with regard to jurisdictional claims in published maps and institutional affiliations.

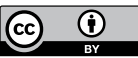

Open Access This article is licensed under a Creative Commons Attribution 4.0 International License, which permits use, sharing, adaptation, distribution and reproduction in any medium or format, as long as you give appropriate credit to the original author(s) and the source, provide a link to the Creative Commons license, and indicate if changes were made. The images or other third party material in this article are included in the article's Creative Commons license, unless indicated otherwise in a credit line to the material. If material is not included in the article's Creative Commons license and your intended use is not permitted by statutory regulation or exceeds the permitted use, you will need to obtain permission directly from the copyright holder. To view a copy of this license, visit http://creativecommons.org/licenses/by/4.0/.

(C) The Author(s) 2020 